\title{
ON THE ZEROS OF POWER SERIES WITH HADAMARD GAPS
}

\author{
W. H. J. FUCHS*
}

Dedicated to KIyoshI Noshiro on his 60 th birthday

1. Let

$$
f(z)=c_{n}+\sum_{k=1}^{\infty} c_{k} z^{n_{k}}
$$

be a power series with Hadamard gaps,

$$
n_{k+1} / n_{k} \geq q>1 \quad(k \geq 1),
$$

convergent in $|z|<1$.

In $1963 \mathrm{G}$. and $\mathrm{M}$. Weiss [1] proved that $f(z)$ assumes every value infinitely often in $|z|<1$, if the constant $q$ in (2) satisfies

$$
q>q_{0}(\approx 100)
$$

and

$$
\sum\left|c_{k}\right|=\infty
$$

In $1964 \mathrm{Ch}$. Pommerenke [2] showed that $f(z)$ assumes every value, at least once, if (2) holds for some $q>1$ and

$$
\lim \sup _{k \rightarrow \infty}\left|c_{k}\right|>0 .
$$

The purpose of this paper is the proof of

THEOREM 1. Let $f(z)$ be given by (1), let (2) be satisfied for some $q>1$ and suppose that (3) holds.

Then $f(z)$ assumes every value infinitely often in $|z|<1$.

2. The proof is based on a lemma whose simplest form $(p=1)$ was already used by G. and M. Weiss and on the idea, due to Hardy and Littlewood, of accentuating the dominance of the largest term of the series (1) by successive

Received June 15, 1966.

* The author gratefully acknowledges support by the National Science Foundation under grant GP 4087. 
differentiations.

LemMa 1. If $g(\zeta)$ is holomorphic in $|\zeta|<R$ and if, for some positive integer $p$

$$
\left|g^{(p)}(\zeta)\right| \leq M \quad(|\zeta|<R) \text {, }
$$

and

$$
\left|g^{(p)}(0)\right| \geq A>0,
$$

then $g(\zeta)$ assumes in $|\zeta|<R$ every value $w$ lying in the disc

$$
|w-g(0)|<K R^{p} A^{p+1} M^{-p},
$$

where $K$ is a positive number depending only on $p$.

Proof. Replacing $g(\zeta)$ by $(g(R \zeta)-g(0)) R^{-p}$, if necessary, we may suppose that

$$
R=1, g(0)=0 .
$$

By Cauchy's formula for the derivatives of a holomorphic function

$$
\left|g^{(p+k)}(0)\right| \leq k ! M \text {. }
$$

Therefore, if

$$
g(\zeta)=\sum_{n=1}^{\infty} c_{n} \zeta^{n} \quad(|\zeta|<1)
$$

then, for $n>p$,

$$
\begin{aligned}
& \left|c_{n}\right|=\frac{1}{n !}\left|g^{(n)}(0)\right| \leq \frac{(n-p) !}{n !} M \leq \frac{M}{p+1}, \\
& \sum_{n=p+1}^{\infty}\left|c_{n}\right| r^{n} \leq \frac{M}{p+1} \frac{r^{p+1}}{1-r} \quad \quad(0 \leq r<1) .
\end{aligned}
$$

By a well-known Lemma on polynomials, due to H. Cartan, for every $\alpha>0$

$$
|P(\zeta)|=\left|\frac{1}{c_{p}} \sum_{n=1}^{p} c_{n} \zeta^{n}\right|>\alpha^{p}
$$

outside circles the sum of whose diameters is less than $4 e \alpha$. Therefore, if

$$
\alpha \leq(1 / 10 e)
$$

it is possible to find an $r$,

$$
\alpha<r<5 e \alpha \leq \frac{1}{2}
$$

such that on the whole circle $|\zeta|=r$

$$
\left|\sum_{n=1}^{p} c_{n} \zeta^{p}\right|=\left|c_{p}\right||P(\zeta)|>\frac{1}{p !} A \alpha^{p}
$$


By (4), (6) and (5), on $|\zeta|=r$,

$$
\begin{aligned}
|g(\zeta)| & >\left|c_{p}\right||P(\zeta)|-\sum_{n=p+1}^{\infty}\left|c_{n}\right| r^{n} \\
& >\frac{1}{p !} A \alpha^{p}-\frac{M}{p+1} \cdot \frac{r^{p+1}}{1-r} \\
& >\frac{1}{p !} A \alpha^{p}-\frac{2 M}{p+1}(5 e)^{p+1} \alpha^{p+1}
\end{aligned}
$$

with the choice

$$
\alpha=\frac{1}{2(p-1) !}(5 e)^{-p-1}(A / M)
$$

(7) proves the existence of a circle $|\zeta|=r$ on which

$$
|g(\zeta)|>K A^{p+1} M^{-p}
$$

and the Lemma follows from (8) by Rouché's Theorem.

LEMMA 2. Let $\left\{n_{k}\right\}_{k=1}^{\infty}$ be a sequence of positive integers satisfying (2). Let $p$ and $\nu$ be positive integers. Put

$$
s_{0}=\exp \left\{-p / n_{\nu}\right\}, s_{1}=\exp \left\{-\frac{p}{2 n_{\nu}}\left(1+\frac{\log q}{q-1}\right)\right\} .
$$

Let

$$
s_{0}<s<s_{1}
$$

and write

$$
w_{k}=n_{k}\left(n_{k}-1\right) \cdots\left(n_{k}-p+1\right) s^{n_{k}}
$$

Then it is possible to find a $p_{0}$ depending only on $q$ such that for $p>p_{0}$ and all large $\nu$

$$
w_{\nu}>4 \sum_{k \neq \nu} w_{k}
$$

Proof. If $n_{k}<p$, then

(9)

$$
w_{k}=0
$$

If $p<n_{k}<n_{\nu}$, then

$$
w_{k} / w_{k+1}<\left(n_{k} / n_{k+1}\right)^{p} s^{-n_{k+1}+n_{k}}<\left(n_{k} / n_{k+1}\right)^{p} s_{0}^{-n_{k+1}+n_{k}}
$$

since

$$
\frac{n_{k}-x}{n_{k+1}-x} \leq \frac{n_{k}}{n_{k+1}} \quad\left(0 \leq x<n_{k}\right) .
$$

Substituting the valne of $s_{0}$ and noting (2), 


$$
\begin{gathered}
w_{k} / w_{k+1}<\sup _{0<t<1 / q} \exp \{p(1-t+\log t)\} \\
\leq \exp \left\{p\left(1-q^{-1}-\log q\right)\right\}
\end{gathered}
$$

Since

$$
\log q=\int_{1}^{q} t^{-1} d t>(q-1) / q
$$

the right hand side of $(10)$ can be made less than $1 / 10$ by choosing

$$
p>\frac{\log 10}{\log q-1+q^{-1}}=p_{1}(q) .
$$

By (9) and (10), if $p>p_{1}(q)$,

$$
\sum_{k<\nu} w_{k}<w_{\nu} \sum_{n=1}^{\infty} 10^{-n}=\frac{1}{9} w_{\nu}
$$

If $k \geq \nu>\nu_{0}(p)$, then

$$
\begin{gathered}
n_{k+1}-x \\
n_{k}-x
\end{gathered}<2^{1 / p} n_{k+1} \quad(0 \leq x \leq p)
$$

Therefore, for all large $\nu$ and $k \geq \nu$

$$
\begin{aligned}
w_{k+1} / w_{k} & <2\left(n_{k+1} / n_{k}\right)^{p} s^{n_{k+1}-n_{k}} \\
& <2\left(n_{k+1} / n_{k}\right)^{\not}\left(s_{1}^{n_{k}}\right)^{\left(n_{k+1} / n_{k}\right)-1} \\
& \leq 2 u^{\not}\left(s_{1}^{n_{\nu} / p}\right)^{p u-p}=\varphi(u),
\end{aligned}
$$

where

$$
u=n_{k+1} / n_{k} \geq q .
$$

In $u \geq q, \varphi(u) \leq \varphi(q)$, Since

$$
\begin{aligned}
\frac{1}{p} \frac{d}{d u} \log \varphi(u) & =\frac{1}{u}-\frac{1}{2}\left(1+\frac{\log q}{q-1}\right) \\
& =\frac{1}{2(q-1)}\left(\frac{(2-u)(q-1)}{u}-\log q\right) \\
& \leq \frac{1}{2(q-1)}\left(\frac{(q-1)}{q}-\log q\right)<0 \quad(u \geq q)
\end{aligned}
$$

Therefore, if $\nu$ is sufficiently large,

$$
\begin{aligned}
w_{k+1} / w_{k} \leq \varphi(q) & =2 \exp \left\{-\frac{1}{2} p(q-1-\log q)\right\} \\
& \leq \frac{1}{10} \quad(k \geq \nu),
\end{aligned}
$$

provided 


$$
p>\frac{2 \log 20}{q-1-\log q}=p_{2}(q)
$$

Then

$$
\sum_{k>\downarrow} w_{k}<w_{\nu} \sum_{n=1}^{\infty} 10^{-n}=w_{\nu} / 9 .
$$

The Lemma now follows with

$$
p_{0}=\max \left(p_{1}(q), p_{2}(q)\right)
$$

from (11) and (13).

3. Proof of Theorem 1. Put

$$
\lim \sup _{k \rightarrow \infty}\left|c_{k}\right|=U .
$$

If $U<\infty$, let $N$ be the least integer such that

$$
\left|c_{k}\right|<\frac{3}{2} U \quad(k>N)
$$

If $U=\infty$, set $N=0$.

Let

$$
\mu(r)=\sup _{k>N}\left|c_{k}\right| r^{n_{k}} \quad(0 \leq r<1) .
$$

Let $\nu=\nu(r)$ be the largest integer such that

$$
\left|c_{\nu}\right| r^{n_{\nu}}>\frac{1}{2} \mu(r)
$$

Notice that

$$
\left|c_{\nu}\right| r^{n_{\nu}}>1\left(r>r_{0}\right), \text { if } U=\infty
$$

$$
\left|c_{\nu}\right| r^{n_{\nu}}>\frac{1}{3} U\left(r>r_{0}\right) \text {, if } U<\infty
$$

Note also that $\nu(r) \rightarrow \infty$ as $r \rightarrow 1-0$.

This is obvious, if $U=\infty$, so that $\mu(r)$ is unbounded. But it is also true for $U<\infty$, since for some arbitrarily large $k$,

$$
\left|c_{k}\right|>\frac{3}{4} U>\frac{1}{2} \mu(r) \quad(r<1) .
$$

We want to show that for any complex number $c$ and any $\rho, 0<\rho<1$,

$$
f(z)=c
$$

has a solution in $\rho<|z|<1$. Changing $f(z)$ into $f(z)-c$, if necessary, it is enough to consider the solutions of 


$$
f(z)=0 \text {. }
$$

The assertion is certainly true, if $f(z)$ has infinitely many zeros in $|z|<1$. We shall now prove it under the (untenable) assumption that $f(z)$ has only a finite number of zeros in $|z|<1$.

Choose $p \geq \max \left(N, p_{0}\right)$, where $p_{0}$ is the constant occurring in Lemma 2. Now choose $r$ so close to 1 that, with the notation of Lemma 2,

$$
\rho<r s_{0}=r e^{-p / n_{\nu}} \quad(\nu=\nu(r)) .
$$

This is possible, since $\nu(r)$, and so also $n_{\nu}$, tends to $\infty$ as $r \rightarrow 1$. Consider $f^{(p)}\left(r s e^{i \theta}\right)$, where, as in Lemma $2, s_{0} \leq s \leq s_{1}$. By Lemma 2,

$$
\begin{aligned}
& \sum_{k \neq \nu} n_{k}\left(n_{k}-1\right) \cdots\left(n_{k}-p+1\right)\left|c_{k}\right|(r s)^{n_{k}} \\
\leq & \sup _{k>N}\left(\left|c_{k}\right| r^{k}\right) \sum_{k \neq \nu} n_{k}\left(n_{k}-1\right) \cdots\left(n_{k}-p+1\right) s^{n_{k}} \\
< & 2\left|c_{\nu}\right| r^{n_{\nu}} \cdot \frac{1}{4} n_{\nu}\left(n_{\nu}-1\right) \cdots\left(n_{\nu}-p+1\right) s^{n_{\nu}} .
\end{aligned}
$$

Hence

$$
\begin{aligned}
& f^{(p)}\left(r s e^{i \theta}\right)=\sum_{k=1}^{\infty} n_{k}\left(n_{k}-1\right) \cdots\left(n_{k}-p+1\right) c_{k}\left(r s e^{i \theta}\right)^{n_{k}-p} \\
= & n_{\nu}\left(n_{\nu}-1\right) \cdots\left(n_{\nu}-p+1\right) c_{\nu}\left(r s e^{i \theta}\right)^{n_{\nu}-p}+E, \\
& |E|<\frac{1}{2} n_{\nu}\left(n_{\nu}-1\right) \ldots\left(n_{\nu}-p+1\right)\left|c_{\nu}\right|(r s)^{n_{\nu}-p} .
\end{aligned}
$$

Now we apply Lemma 1 to

$$
g(\zeta)=f\left(r\left(s_{0} s_{1}\right)^{1 / 2} e^{\zeta+i \theta}\right) .
$$

In view of (15) $g(\xi)$ satisfies the hypothesis of Lemma 1 with

$$
\begin{aligned}
R & =\frac{1}{4}\left(1+\frac{\log q}{q-1}\right) \frac{p}{n_{\nu}} \\
M & \leq / K_{1}(p) n_{\nu}^{p} \mid c_{\nu} !\left(r s_{1}\right)^{n_{\nu}} \quad\left(n_{\nu}>2 p\right) \\
A & \geq / K_{2}(p) n_{\nu}^{p}\left|c_{\nu}\right|\left(r s_{0}\right)^{n_{\nu}} .
\end{aligned}
$$

Therefore, by (14), for all $r>r_{0}$,

$$
\begin{aligned}
& R^{p} A^{p+1} M^{-p}>C_{1}(p, q)\left|c_{\nu}\right|\left(r s_{0}\right)^{n_{\nu}}\left(s_{0} / s_{1}\right)^{n_{\nu} p} \\
> & C_{2}(p, q)\left|c_{\nu}\right| r^{n_{\nu}} \\
> & C_{3}
\end{aligned}
$$

where $C_{3}$ depends only on $p, q$ and $U$.

The conclusion of Lemma 1 for our choice of $g(\zeta)$ asserts that $f(z)$ takes on every value $w$ in the disc 


$$
\left|w-f\left(r\left(s_{0} s_{1}\right)^{1 / 2} e^{i \theta}\right)\right|<C,
$$

where $C$ depends only on $p, q$ and $U$.

In particular $f(z)$ will take on the value 0 in $r>r_{0}$, if for some $r$ arbitrarily close to 1

$$
\left|f\left(r\left(s_{0} s_{1}\right)^{1 / 2} e^{i \theta}\right)\right|<C .
$$

Suppose that (18) were not the case. We shall derive a contradiction with the aid of the first fundamental Theorem of the Nevanlinna Theory. Since $f(z)$ has only a finite number of zeros in $|z|<1$,

$$
N(t, 1 / f)=0(1) . \quad(t<1)
$$

If $(18)$ is not true for $r>r_{0}$, then

$$
\begin{aligned}
& m(t, 1 / f)=\frac{1}{2 \pi} \int_{0}^{2 \pi} \log ^{+}\left|1 / f\left(t e^{i \theta}\right)\right| d \theta \\
< & \log ^{+}(1 / C) .
\end{aligned}
$$

Therefore, by the First Fundamental Theorem

$$
\begin{aligned}
m(t, f) & =m(t, 1 / f)+N(t, 1 / f)+0(1) \\
& =0(1) ;
\end{aligned}
$$

That is to say that the function $f(z)$ is of bounded Nevanlinna characteristic. Then

$$
\lim _{r \rightarrow 1} f\left(r e^{i \theta}\right)
$$

exists for almost all $\theta$ (3, Th. 7.25, p. 276). But, by (3),

$$
\sum_{k}\left|c_{k}\right|^{2}=\infty
$$

This implies that the radial limit (19) does not exist for almost all $\theta$ (3, Th. 6.4 , p. 203).

Therefore $m(t, f)$ (and so $m(t, 1 / f)$ ) must be unbounded and (18) holds. This completes the proof of Theorem 1 .

4. The result of $\mathrm{G}$. and $M$. Weiss quoted in the introduction makes it likely that condition ( 3 ) of Theorem 1 could be replaced by

$$
\sum_{k}\left|c_{k}\right|=\infty
$$

I have not been able to prove this. 
It would also be interesting to know whether every sector $\alpha<\arg z<\beta$ contains zeros of $f(z)$ under the hypothesis of Theorem 1.

It is a pleasure to acknowledge the help of Dr. Linda Sons in the preparation of this paper.

\section{REFERENCES}

[1] G. Weiss and M. Weiss, On the Picard property of lacunary power series, Studia Math. 22 (1963), 221-245.

[2] Ch. Pommerenke, Lacunary power series and univalent functions, Michigan Math. J. 11 (1964), 219-233.

[3] A. Zygmund, Trigonometric Series, Vol 1, Cambridge University Press

Cornell University

Ithaca, New York 